\title{
Operating Experience with High Beta Superconducting RF Cavities*
}

\author{
H. F. Dylla, L. R. Doolittle and J. F. Benesch \\ Continuous Electron Beam Accelerator Facility \\ 12000 Jefferson Avenue, Newport News, VA 23606-1909 USA
}

\begin{abstract}
The number of installed and operational $B=1$ superconducting If cavities has grown significantly over the last two years in accelerator laboratories in Europe, Japan and the U.S. The total installed acceleration capability as of mid-1993 is approximately $1 \mathrm{GeV}$ at nominal gradients. Major installations at CERN, DESY, KEK and CEBAF have provided large increments to the installed base and valuable operational experience. A selection of test data and operational experience gathered to date is reviewed.
\end{abstract}

\section{INTRODUCTION}

Superconducting Radio-Frequency (SRF) cavities for speed of light particles $(B=1)$ are playing an increasing role in high energy and nuclear physics accelerators worldwide. As more cavities are manufactured, tested, and put into service, the breadth and depth of operational experience is growing. Installed acceleration capacity is approximately $1 \mathrm{GeV}$ and a total of about $10^{6}$ cavity-hours of operation has been accumulated. Figure 1 shows the time history of installed SRF acceleration capacity.

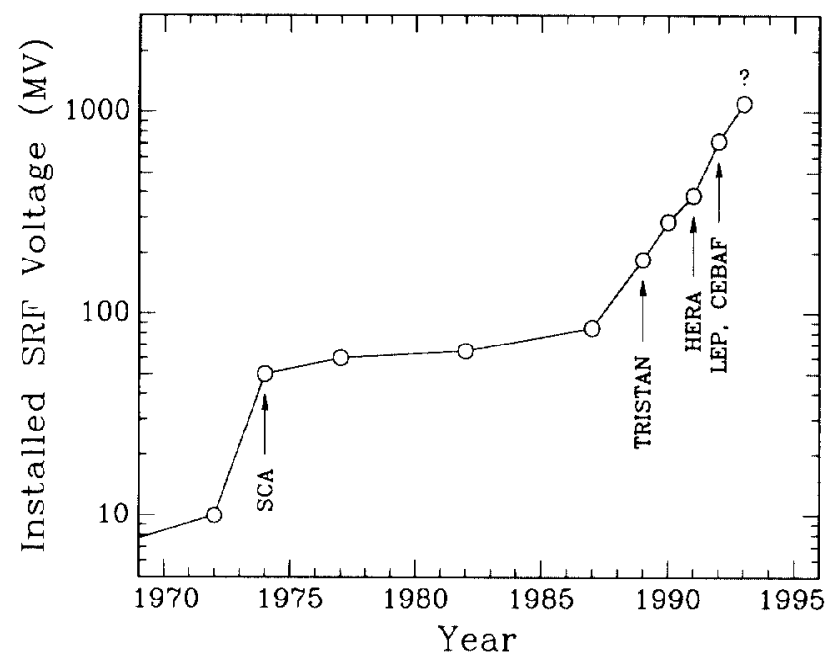

Figure 1. History of installed SRF acceleration capacity

The range of parameters spanned by the cavities in use is substantial. Cavities are constructed either of solid niobium or niobium sputtered onto formed copper. They are operated between $1.8 \mathrm{~K}$ and $4.5 \mathrm{~K}$. Frequencies range from $352 \mathrm{MHz}$ to $3000 \mathrm{MHz}$. Beam currents range up to $70 \mathrm{~mA}$. Gradients in laboratory tests of production cavities range from 5 to 20 $\mathrm{MV} / \mathrm{m}$. Once these cavities are installed in cryostat systems, additional constraints due to the system interlocks and the interfaces result in system gradients ranging from 3 to 12 $\mathrm{MV} / \mathrm{m}$.

*This work supported by US DOE Contract DE-AC05-84ER401540
Several accelerator SRF projects are complete and operational: Stanford's HEPL, KEK's TRISTAN, Darmstadt's S-Dalinac, DESY's HERA, and CERN's SPS. Accelerator projects using SRF that are under construction and have some operational experience are CEBAF, CERN's LEP, Saclay, and Frascati. Possible future applications include TESI.A [1.3], spallation neutron sources and FEL drivers. See also Table 1.

This paper presents a summary of present performance and experience of the installations worldwide. Topics include operational aspects of the cavities and their peripheral hardware. Particular emphasis is placed on CEBAF's experience with SRF cavities because the authors are closest to it and because the number of units at CEBAF is about $80 \%$ of those now installed worldwide.

\section{OPERATIONAL EXPERIENCE WITH SRF}

\section{A. Tristan (KEK)}

This system comprises thirty-two $508 \mathrm{MHz}$ cavities installed in pairs in 16 cryostats and operated at 4.5K.[1] Total active length is $47.2 \mathrm{~m}$. In pre-operational testing, the average gradient was $7 \mathrm{MV} / \mathrm{m}$, while in operation this drops to 3.2-4.7 MV/m. 400,000 cavity-hours of operation with beam have been accumulated. Operational problems at startup included vacuum leaks at beam pipe indium joints, since solved, and coaxial coupler ceramic punch throughs. There have been some difficulties with the piezo-electric devices used to tune the cavity frequency within the cryostat. A few cavities experienced arc trips associated with synchrotron radiation; these were reduced when intercepts were installed.

\section{B. DESY}

The HERA electron ring includes sixteen $500 \mathrm{MHz}$ cavities in eight cryostats, totaling 19.2 active meters. The operating temperature is $4.4 \mathrm{~K}$. In pre-operational testing, the average gradient was $6 \mathrm{MV} / \mathrm{m}$, while in operation this drops to $4 \mathrm{MV} / \mathrm{m}$. 160,000 cavity-hours of operation with beam have becn accumulated. Operational problems at startup included $Q$ degradation with slow cooldown.[2] This "Q-disease" was determined to be due to hydride formation in the temperature range $70-170 \mathrm{~K}$. A fast cooldown from $170 \mathrm{~K}$ to below $70 \mathrm{~K}$ eliminates the difficulty, but this can be difficult for some machines due to cryogenic system limitations. [3] Raising the temperature to $200 \mathrm{~K}$ for two hours redistributes the hydrogen and $Q$ is often restored. [4] High temperature heat treatment above $800{ }^{\circ} \mathrm{C}$ eliminates the problem and in laboratory experiments anodization of the niobium surface considerably reduced the Q-degradation. $[3,5]$

\section{Darmstadt}

S-Dalinac includes ten and one quarter $3000 \mathrm{MHz} 20$-cell cavities in two cryostats, totaling 10.25 active meters.[6] (Fractional cavities have fewer than the standard number of cells for a given machine.) The operating temperature is $1.8 \mathrm{~K}$. In operation, the average gradient is $5.6 \mathrm{MV} / \mathrm{m}$. 
43,000 cavity-hours of operation with beam have been accumulated. Some of the cavities have lower $Q$ than expected. This, coupled with refrigeration limitations, has led the system to be operated at less than design energies. Replacement cavities are due to be installed shortly.

\section{Saclay}

MACSE includes five $1500 \mathrm{MHz}$ cavities in two and a half cryostats, totaling 2.5 active meters, operating at $1.8 \mathrm{~K} .[7]$ (Fractional cryostats have fewer than the usual number of cavities for a given machine.) In opcration the average gradient is $12 \mathrm{MV} / \mathrm{m}$. Operational problems at startup included a cold sapphire of window failure.

Saclay has made great strides in closed loop chemical treatment and cleaning systems for SRF cavities. Results have been excellent, with gradients on test cavities ranging to 28 $\mathrm{MV} / \mathrm{m} .[8]$

\section{E. CERN}

LEP 200 is the on-going upgrade of the LEP accelerator system with SRF cavities to double the available particle energy. The installation will consist of 192 cavities in 48 cryostats operating at $352 \mathrm{MHz}$.[9] The operating temperature is $4.5 \mathrm{~K}$. As of May 1993, twelve cavities in three cryostats have been installed and operated with beam. This represents $20.4 \mathrm{~m}$ of active length, about $6 \%$ of the total planned $(326 \mathrm{~m})$. In pre-operational testing, the average gradients were 5-6 $\mathrm{MV} / \mathrm{m}$, while in operation this drops to $3.5-5.5 \mathrm{MV} / \mathrm{m}$. By contract, the vendors are allowed $20 \%$ maximum degradation between vertical test and tunnel horizontal cryostat test results. 30,000 cavity-hours of operation with beam have been accumulated. Startup problems include difficulties with coaxial input power couplers and HOM output couplers.

\section{F. CEBAF}

CEBAF now has 113 active meters installed for operation at $2 \mathrm{~K}$; upon completion in December 1993 it will comprise 169 active meters. The machine will include 338 cavities operating at $1497 \mathrm{MHz}$ in $421 / 4$ cryostats. In pre-operational testing, the average gradients at field emission onset are 7.5$10 \mathrm{MV} / \mathrm{m}$, while in operation in the horizontal cryostat usable gradient is $5-7.5 \mathrm{MV} / \mathrm{m} .140,000$ cavity-hours of operation with beam have been accumulated.[10] One cryomodule was pushed to an average gradient of $8 \mathrm{MV} / \mathrm{m}$ with beam. Operational problems at startup include system performance limited by refrigeration capacity until the $2 \mathrm{~K}$ cold compressor system functions (expected in late 1993). It appears that consistent system operation above $7.5 \mathrm{MV} / \mathrm{m}$ will require of conditioning of the waveguide transition from room temperature to $2 \mathrm{~K}$ and better understanding of the interactions among the hardware interlocks monitoring performance.

\section{CURRENT STATE OF THE ART}

Significant advances are being made in pushing cavity performance, as reported in several papers at this conference. Proch reviewed this area in these proceedings, [11], so only highlights will be given here. More work is needed: the usable gradient assumed for the contemplated European Electron Machine is $10 \mathrm{MV} / \mathrm{m}$ at $\mathrm{Q} \geq 4 \times 10^{9}$.[12] The usable gradient goal for the cavities being fabricated for the TESLA
Test Facility is $15 \mathrm{MV} / \mathrm{m}$ at $\mathrm{Q} \geq 5 \times 10^{9}$. TESLA itself will require a usable gradient of $25 \mathrm{MV} / \mathrm{m}$ at the same Q. [13]

The best production (5-cell) cavity at CEBAF reached 20 $\mathrm{MV} / \mathrm{m}$ gradient in the vertical test dewar. No special care was taken in preparing this cavity; it represents the outlier of the distribution. This result is shown in Figure 2. One cavity in the CEBAF production run was specially treated, and achieved $20.5 \mathrm{MV} / \mathrm{m}$. On initial testing (standard processing) this latter cavity reached $16 \mathrm{MV} / \mathrm{m}$ without field emission. It was subsequently annealed at $1400^{\circ} \mathrm{C}$ in an UHV furnace with titanium as a solid state getter, chemically etched and rinsed with water at moderate pressure (10 MPa). [14] Cornell reported at this conference results on a 9 cell cavity at $3 \mathrm{GHz}$ which achicved $30 \mathrm{MV} / \mathrm{m}$.[15] For CEBAF, the surface field is 2.56 times the accelerating field, while the ratio is $2.1: 1$ for the Cornell cavity.

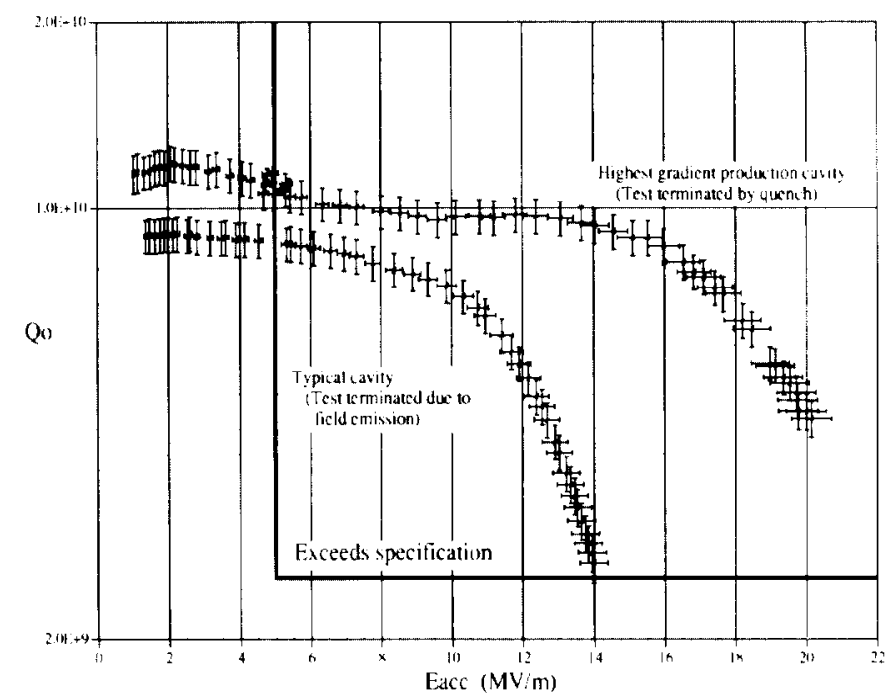

Figure 2. Performance of two CEBAF cavities

\section{CEBAF CAVITY TESTING}

At CEBAF, the basic unit of the accelerating structure is a hermetically sealed pair of cavities. The cavities are chemically etched, rinsed with high purity water and alcohol (isopropanol or methanol), assembled in a class 100 clean room with the necessary auxiliary hardware, and evacuated; all within a five hour period. The cavity pairs remain under vacuum thereafter. Each cavity pairs is hung from a dewar lid, connected to variable coaxial couplers, and subjected to a full rf performance test at $2 \mathrm{~K}$ in a vertical dewar. This has proved advantageous compared to procedures during which cavities have to be exposed to atmosphere after initial testing, raising the chances of introduction of particulate contamination. .

As of May 1, 1993, 250 cavities had been assembled into pairs and tested. CEBAF defines usable gradient as the lower of the gradient with $1 \mathrm{~W}$ of field emission dissipation or the quench gradient less $1 \mathrm{MV} / \mathrm{m}$. Using this definition, the average usable gradient achieved was $9.4 \mathrm{MV} / \mathrm{m}$ at $\mathrm{Q}=7 \times 10^{9}$ versus $5 \mathrm{MV} / \mathrm{m}$ at $2.4 \times 10^{9}$ specified. In vertical test, gradient was limited by the following mechanisms: $5 \%$ quench without any field emission, $35 \%$ quench with field emission and $60 \%$ field emission sufficient to excecd 
available if power $(80 \mathrm{~W})$ or a self-imposed radiation limit. In Figure 2 we show the $Q$ versus $E$ plots of two cavities, a typical unit with field emission loading at moderate gradients and the best production cavity to date, with field emission beginning at $12 \mathrm{MV} / \mathrm{m}$ and a thermal-magnetic quench at $20.1 \mathrm{MV} / \mathrm{m}$ with $46 \mathrm{~W}$ power dissipation, about half in field emission. In Figure 3 we show field emission onset gradients versus time. It is noteworthy that the standard deviation of the field emission onset gradient and usable gradients has not changed since production began: $\sigma=3 \mathrm{MV} / \mathrm{m}$ has remained constant while the average gradients increased by $50 \%$. The source of this performance spread is not understood. A cavity fabrication and performance database is being assembled for further trending and feedback to cavity performance, but no variable examined to date has correlated well with the width of the distribution. This effort will continue after the cavity pair production is complete to provide information to the accelerator community.

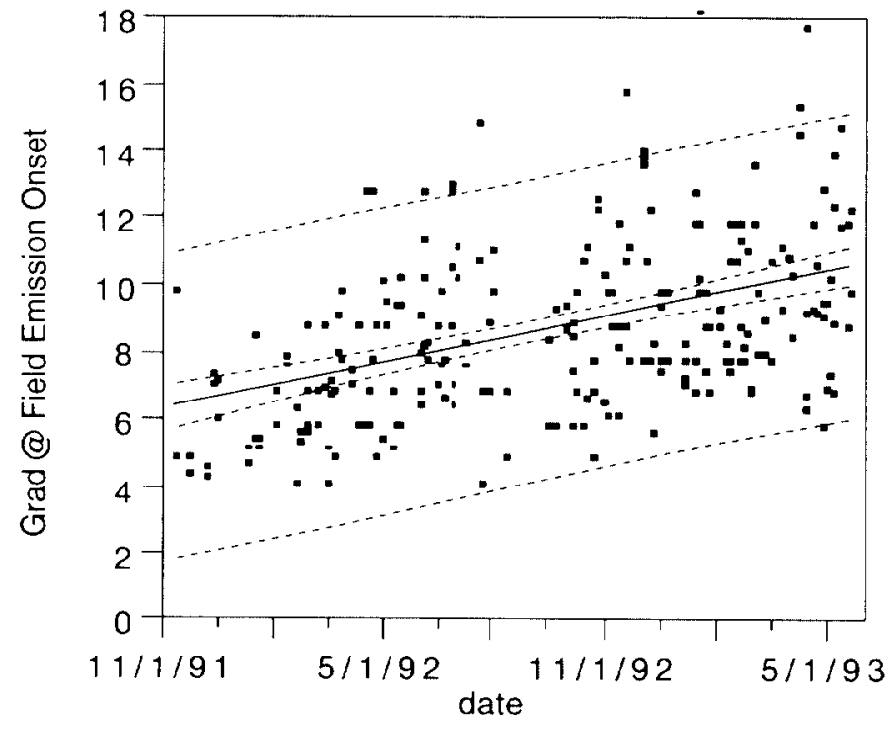

Figure 3. Gradient at field emission onset versus time for CEBAF cavity production

The cavities which did quench without field emission did so with a spread in gradients of 8 to $18 \mathrm{MV} / \mathrm{m}$. This is sufficient data to indicate that the niobium used in the CEBAF cavities, with $R R R=250$ as supplied and $R R R \approx 200$ as welded, does not yet provide the thermal stability needed for $\mathrm{E}_{\text {acc }}>15$ $\mathrm{MV} / \mathrm{m}$ as desired for future accelerators. Even the titanium gettered cavity cited above, with an RRR $>800$, was limited to $20.5 \mathrm{MV} / \mathrm{m}$. More work on the basic materials used in cavities is clearly needed.

\section{SRF CAVITY FIELD EMISSION LIMITATION}

As mentioned above, only about $5 \%$ of the CEBAF cavities show no field emission loading, i.e., have a flat $Q$ profile until quench. There are two types of electron loading in cavities, resonant (multipacting) and non-resonant (field emission). The first is reasonably well understood and can be eliminated by proper cavity shaping.[16] Non-resonant electron loading is due to both intrinsic and extrinsic impurities of the niobium surface. Field emission loading needs to be limited to minimize radiation damage to coupler ceramics which can result in vacuum leaks and to minimize radiation damage to the niobium itself.

CEBAF has steadily improved its average gradient at field emission onset over time by careful rinsing and attention to clean assembly techniques. The data shows that much improvement is still possible, and many techniques are being investigated in labs around the world. High pressure (10 MPa) water rinsing has shown greal promise at CERN [17] and CEBAF [14]. Cornell has also successfully applied high power pulsed processing to eliminate (by explosion) field emission sites.[18] Cornell [19] and Wupertal [20] have shown that heat treatment in a UHV furnace above $1200{ }^{\circ} \mathrm{C}$ can also reduce field emission. Of these techniques, the high pressure rinsing is the easiest to implement and therefore the most likely to enter manufacturing service soon.

\section{SRF PERFORMANCE LIMITATIONS IN ACCELERATORS}

One has to make a clear distinction between the fundamental capability of the SRF cavity technology and its practical application in an accelerator environment. For the group of people dealing with cavities alone, it is important to stretch the performance of a cavity to its limit. The results of such tests, generally done under well controlled conditions in a vertical configuration without beam, point towards fundamental performance limitations: anomalous losses caused by defects, thermal-magnetic breakdown, or field emission loading. In the accelerator environment, often the prior performance of the cavities cannot be repeated due to external constraints which are not present in the well controlled vertical tests. Among these are power handling problems ( $\mathrm{rf}$ heating and outgassing) in external waveguides (CEBAF), rf power limitations due to low $Q$ external (CEBAF), insufficient masking of synchrotron radiation (KEK), limitations in cryogenics due to high rf losses ( $Q$ disease, HERA) and nun-performing (incomplete) cryosystems (CEBAF).

At CEBAF, there are several interlocks installed in the cavity cryostats to protect the system. At the present time, since there is little operating experience, these are set very conservatively. Most of these monitor the waveguide between the warm and cold rf windows. There is a phototube which senses the light from arcs in the waveguide. If light is seen for more than $50 \mu \mathrm{s}$, the $\mathrm{rf}$ is shut off. There is a thermopile which monitors the IR radiation in the waveguide. If this sensor goes above a set point which corresponds to about $50{ }^{\circ} \mathrm{C}$ on a warm polyethylene window, the if is shut off. The ion pump on this waveguide section is monitored and the of shut off if the pressure exceeds $10^{-7}$ torr. Finally, the cavity is administratively limited to $1 \mathrm{~W}$ of field emission loading as measured calorimetrically. With all these constraints, the first 12 cryomodules at CEBAF (96 cavities) averaged $7.2 \mathrm{MV} / \mathrm{m}$ usable gradient versus a specification of $5 \mathrm{MV} / \mathrm{m}$. Within the allowed dynamic heat load of $45 \mathrm{~W}$, the average gradient is $6.5 \mathrm{MV} / \mathrm{m}$. Cavity performance has continued to improve and these values are expected to increase for the entire machine when installed and commissioned. Further increases are expected as the waveguide is conditioned and the machine is better understood, allowing some interlocks to be relaxed. 
Net performance of a cavity necessarily diminishes as more and more requirements and support equipment are considered. This has little to do with actual degradation of the cavity, and more to do with the fact that a chain is only as strong as its weakest link. We note in passing that the design specification for CEBAF cavity operation is $5 \mathrm{MV} / \mathrm{m}$ with $\mathrm{Q}_{0} \geq 2.4 \times 10^{9}$, and that neither typical cavities nor their supporting hardware has any problems operating at that level. Subsystems and infrastructure start to show their limits when asked to perform at $150 \%$ of specifications and higher, although the CEBAF cavities (on average) are capable of operation at about $200 \%$ of specifications. In Figure 4 we compare vertical test performance with tunnel commissioning performance for 125 cavities, while in Figure 5 we remove the 47 cavities constrained in the tunnel by interlocks from the comparison.

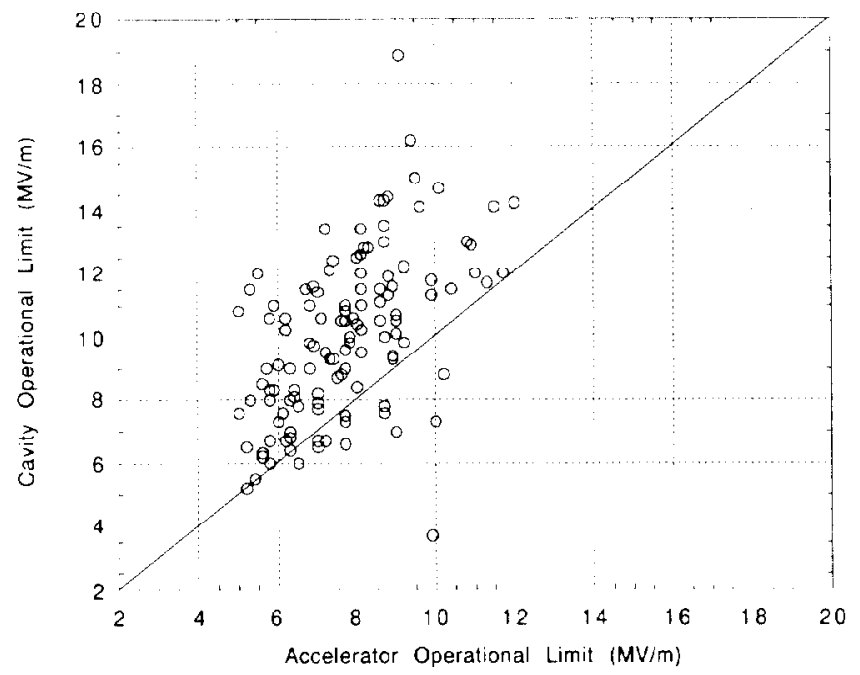

Figure 4. Comparison of vertical test with accelerator limit derived all constraints on tunnel commissioning tests

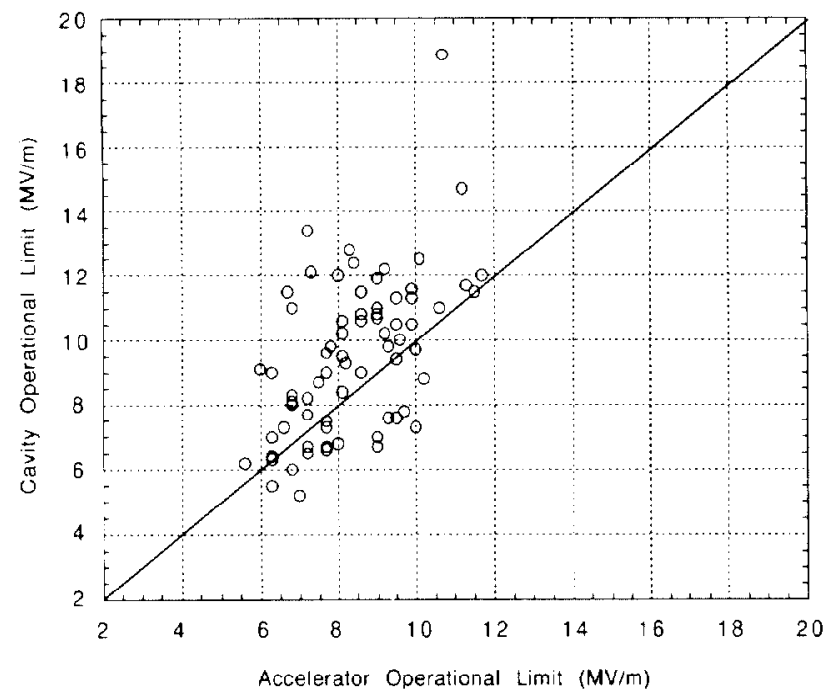

Figure 5 Comparison of vertical test with accelerator limit derived from only those constraints on tunnel operation which are also present in vertical test.

\section{HIGHER ORDER MODE POWER EXTRACTION}

Higher order modes (HOMs) must be adequately damped to prevent various forms of beam breakup. Different accelerators have different requirements, and the problems increase with increasing beam current, decreasing rf bucket length, increasing number of passes, and decreasing time between bunches.

High current machines such as storage rings generate large amounts of HOM power. TRISTAN, in fact, has problems with power handling on the HOM output coupler, and has had to replace the couplers. Low current machines like CEBAF, on the other hand, will only dissipate a fraction of a watt of HOM power $(0.25 \mathrm{~W}$ per 5 -cell cavity at a beam current of $1 \mathrm{~mA}$ ). The heat load involved in couplers to take this power out to room temperature or even a thermal shield at 40-80K would exceed the power in the modes themselves, and therefore CEBAF has HOM loads inside the cryostat at $2 \mathrm{~K}$. Materials requirements for these loads were themselves something of a challenge.[21] They must have temperature independent microwave absorption properties, be vacuum compatible, and have good thermal conductivity.

Operational measurements of the damping of HOMs in CEBAF cavities with beam indicate that the beam breakup (BBU) threshold is about 14 ma, which is 70 times the design current. Future SRF accelerator projects, such as CERN LEPII, Cornell B-factory, and FEL drivers, will have HOM damping and power handling requirements more severe than those of accelerators in operation today, and research to advance the technology is underway.[21]

\section{COUPLER CERAMICS}

As mentioned above, several laboratories have had trouble with arcing and breakdown of coupler ceramics, leading to reduced power handling limits or vacuum leaks through the ceramics. As a result, many laboratories are contributing to the knowledge base on breakdown properties of ceramics, in particular high purity alumina. There are at least two distinct phenomena related to the interaction of ionizing radiation with the ceramics. Glass $\left(\mathrm{SiO}_{\mathrm{X}}\right)$ impurities must be minimized because radiation dosage leads to decomposition and formation of vacuoles which increase permeation rates and can eventually link up to form a true leak.[22] Ionizing radiation can also cause surface flashover. The second phenomenon has been seen at CEBAF.[23] These phenomena may be the cause of small $\left(\sim 10^{-8} \mathrm{~atm}-\mathrm{cc} / \mathrm{s}\right)$ vacuum leaks which are infrequently found in the CEBAF cold rf window ceramic after vertical test.

Multipacting has been identified as a source of of window failure and antimultipacting coatings, such as $\mathrm{TiN}, \mathrm{TiO}_{2}$ or $\mathrm{Cr}_{2} \mathrm{O}_{3}$, are routinely applied to ceramic of windows.[24] Coatings with controlled resistivity, designed to provide some charge drainage while minimizing if loss in the coating, are under development. Gold and other impurity dispersions in the antimultipacting coatings cited appear promising.[25] Above all, good surface preparation, cleaning and handling procedures are mandatory. 


\section{CAVITY VACUUM INTEGRITY}

Cavity vacuum integrity has been a problem for the field because of the sensitivity of the indium sealed niobium flanges to surface preparation. A recent technique developed at CEBAF has increased the sensitivity for measuring the vacuum integrity of cavity assemblies.[26,27] Since the cavity pairs are submerged in liquid helium for roughly a day, an integrated leak test provides great sensitivity. In the CEBAF cavity pairs there are 16 indium joints $(4.75 \mathrm{~m})$ and 4 copper knife edge seals. The average integrated leak rate for 80 pairs was $5.8 \times 10^{-11} \mathrm{~atm}-\mathrm{cc} / \mathrm{s}$.

\section{SUMMARY}

Superconducting if technology is finally maturing as a reliable and cost effective means of accelerating high quality particle beams. Significant operational experience is being clocked now and will double in the next year. Performance is also improving, with production cavity gradients of 10-20 $\mathrm{MV} / \mathrm{m}$ in vertical test and $5-12 \mathrm{MV} / \mathrm{m}$ when installed in accelerators and subject to additional systems requirements. Significant progress has been made on key technical issues which can limit performance and reliability, including HOM power extraction, cavity assembly vacuum integrity, and power input couplers. Additional work is needed on the last item as power handling requirements increase to the MW level.

\section{ACKNOWLEDGMENTS}

The authors wish to acknowledge the assistance of the CEBAF SRF and Operations Departments, especially P. Kneisel, M. Drury, C. Reece, W. Schneider, J. Mammosser, K. Hovater and C. Sinclair. B. Aune of Saclay, W. Weingarten of CERN, D. Proch of DESY, H. Graef of Darmstadt and S. Noguchi of KEK provided information used in the laboratory summaries.

\section{REFERENCES}

[1] S. Mitsunobu, et al., Proc. of the 5th Workshop on RF Superconductivity, p 84, DESY M-92-01 (April 1992); $S$. Noguchi, private communication.
[2] G. Enderlein et al., Proc. of the 1991 Particle Accel. Conf, p 2432; and D. Proch, private communication.

[3] B. Bonin and R.W. Roth, ibid, p. 210.

[4] K. Saito and P. Kneisel, Proc. of the 3rd European Particle Accelerator Conference EPAC 92, p. 1231.

[5] K. Saito and P. Kneisel, Proc. of the 5th Workshop on RF Superconductivity, p. 665, DESY M-92-01 (April 1992).

[6] J. Auerhammer, et al., Proc. of the 5th Workshop on RF Superconductivity, p. 110, DESY M-92-01 (April 1992)

[7] B. Aune, private communication.

[8] B. Bonin, these proceedings.

19] C. Wyss, these proceedings; G. Cavallari, et al., these proceedings.

[10] A. Hutton, these proceedings.

[11] D. Proch, these proceedings.

[12] M. Promé, private communication.

[13] H. Edwards, these proceedings.

[14] P. Kneisel and M. Rao, these proceedings.

[15] J. Graber, et al., these proceedings.

[16] C. M. Lyneis, Proc. of the Workshop on RF Superconductivity, p. 119, KfK 3019 (November 1980).

[17] Ph. Bernard, et al., Proc. of the 3rd European Particle Accelerator Conference EPAC 92, p. 1269.

[18] J. Graber, et al., these proceedings.

[19] J. Kirchgessner, et al., Proc. of the 5th Workshop on RF Superconductivity, p. 37, DESY M-92-01 (April 1992) and references therein.

[20] R. W. Roth, et al., Proc. of the 2nd European Particle Accelerator Conference EPAC 90, p.1097 Editiones Frontieres.

[21] I. Campisi, these proceedings.

[22] D. Howitt, Proc. of the Workshop on Microwave Absorbing Materials for Accelerators, February 1993 (to be published).

[23] L. Phillips, et al., these proceedings; T. Powers et al., these proceedings.

[24] A. R. Nyaiesh, et al., J. Vac. Sci \& Tech. A3, 1705 (1986).

[25] L. Phillips, private communication

[26] M. G. Rao, J. Vac. Sci. \& Tech. All (to be published in July/August, 1993).

[27] J. Mammosser and J. F. Benesch, these proceedings.

Table 1 - Machine Parameters

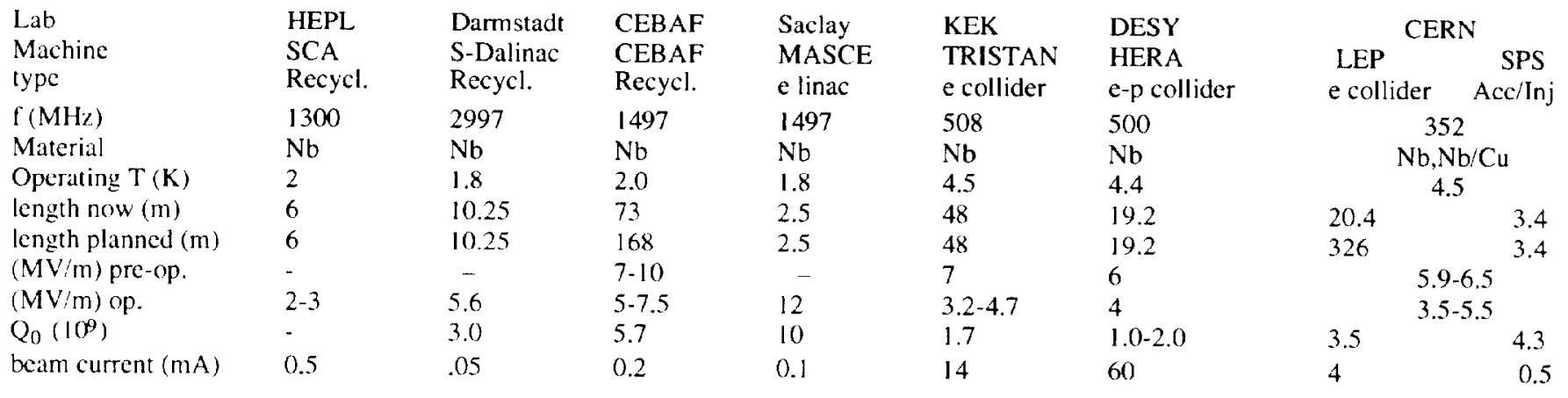

